\title{
Sobre las mujeres desde las historias de las mujeres, Gente de Centro y muchas Otras en diálogo con María<smiles></smiles>

\section{Artículo de investigación}

\section{María José Arbeláez Grundmann}

mjarbelaez@gmail.com

Universidad Distrital Francisco José de Caldas, Bogotá, Colombia.

Recibido: 11 de noviembre de 2018

Aprobado: 10 de junio de 2019

Cómo citar este artículo: (2020). Sobre las mujeres desde las historias de las mujeres, Gente de Centro y muchas Otras en diálogo con María José. Calle 14: revista de investigación en el campo del arte 15(27). pp. 152-167. DOI: https://doi.org/10.14483/21450706.15418

Agradecimientos: Al Centro de Investigaciones y Desarrollo Científico CIDC de la Universidad Distrital F.J.C. por el apoyo al proyecto de investigación-creación "El saber sensible de las mujeres indígenas Gente de Centro recorriendo sus chagras en Araracuara, Caquetá". A las abuelas y los sabedores Alicia Sánchez, Raquel Andoque, Marlene Ramírez, Alcira Sueroque, María Sabina, Nazaret Cabrera, Aidé Barbosa, Patricia Márquez, Juana Suárez, Isolina Simón de Cabrera, Isaías Román, Aurelio Mendoza. Al maestro Andrés Corredor. 


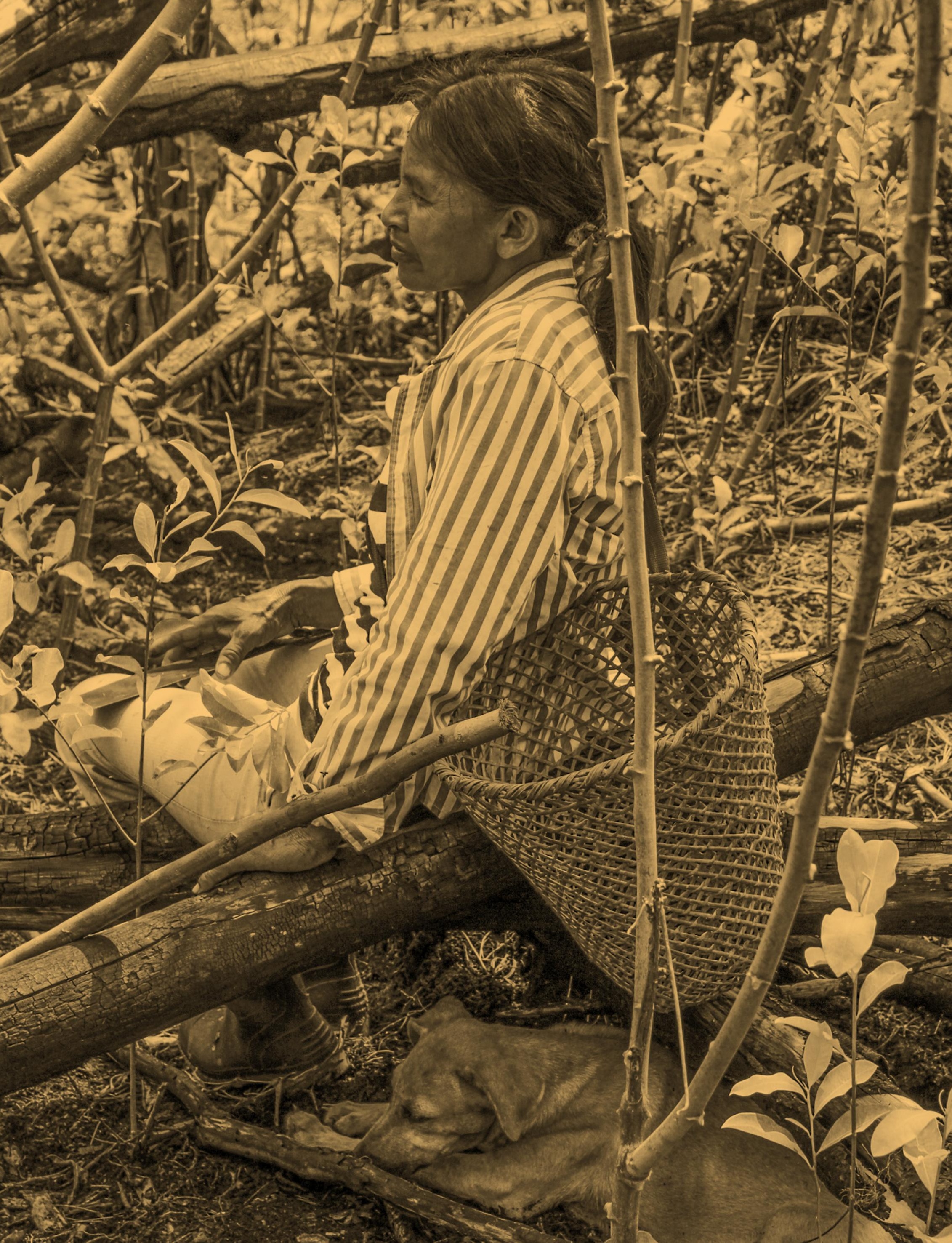


Sobre las mujeres desde las historias de las mujeres, Gente de Centro y muchas otras en diálogo con María José

\begin{abstract}
Resumen
En el presente texto expongo apartes del dialogo sostenido con las abuelas indígenas y sabedores de la Gente de Centro en Araracuara (Caquetá), y con Otras mujeres y hombres que habitan concepciones de mundos espirituales enraizadas en lo vital de las prácticas cotidianas y en el respeto de los seres: ellos nos presentan una alternativa de modos de vida relacional diferente a la acostumbrada de modo hegemónico en Occidente. La cuestión que acucia el tránsito de este camino es el de reconocer los saberes sensibles practicados por las mujeres indígenas de Gente de Centro y de Otras mujeres y hombres comprometidos con los cuidados de la naturaleza y de la familia, creadoras en conexión con la tierra y el cosmos desde la alegría profunda del estar vivas, habitando comunidades humanas equitativas y amorosas.
\end{abstract}

\title{
Palabras Clave
}

Mujeres indígenas; trabajos femeninos; cuidado de la familia; cuidado de la Tierra; Madre Tierra; chagra.

\section{On Women from the Stories of Women, Gente de Centro and Many Others in a Dialogue}

\begin{abstract}
I present here parts of the dialogue I held with the indigenous grandmothers and sabedores of Gente de Centro in Araracuara (Caquetá), and with Other women and men who inhabit conceptions of spiritual worlds rooted in the vitality of everyday practices and the respect for all beings: they show us alternative relational ways of life, very different from those hegemonically prevalent in the West. The issue that drives the transit of this road is to recognize the sensorial knowledge set practiced by the indigenous women of Gente de Centro and Other women and men committed to the care of nature and family, creators who are in connection with the land and the cosmos from a deep joy of being alive, within equitable and loving human communities.
\end{abstract}

\section{Keywords}

Indigenous women; female jobs; family care; Earth care; Mother Earth; chagra

Sur les femmes depuis les histoires de femmes, de Gente de Centro et de beaucoup d'autres en dialogue avec María José

\section{Résumé}

Je présente ici des extraits du dialogue que j'ai eu avec les grands-mères et les sabedores indigènes de Gente de Centro à Araracuara (Caquetá) et avec d'autres femmes et hommes qui habitent des conceptions du monde spirituel enracinées dans la vitalité des pratiques quotidiennes et le respect de tous les êtres : elles nous montrent une alternative de modes de vie relationnels, très différents de ceux prévalant de manière hégémonique en Occident. La question qui anime le transit de cette route est de reconnaître les savoirs sensoriels pratiqués par les femmes indigènes du Gente de Centro et par d'autres hommes et femmes engagées dans la protection de la nature et de la famille, créatrices en lien avec la terre et le cosmos depuis une joie profonde d'être en vie, au sein de communautés humaines équitables et affectueuses.

\section{Mots clés}

Femmes indigènes ; emplois féminins ; soins de la famille ; soins de la terre ; Terre Mère ; chagra 
Sobre as mulheres a partir das histórias das mulheres, Gente de Centro e muitas Outras em diálogo com María José

\section{Resumo}

Apresento aqui partes do diálogo que mantive com as avós indígenas e sabedores do Gente de Centro em Araracuara (Caquetá), e com outras mulheres e homens que habitam concepções de mundos espirituais enraizadas na vitalidade das práticas cotidianas e no respeito a todos os seres. Elas nos mostram uma alternativa de modos de vida relacionais, muito diferentes daqueles hegemonicamente predominantes no Ocidente. A questão que impulsiona o trânsito dessa estrada é reconhecer o conjunto de saberes sensoriais praticados pelas mulheres indígenas de Gente de Centro e outras homens e mulheres comprometidos com o cuidado da natureza e da família, criadoras que estão em conexão com a terra e o cosmos da alegria profunda de estar vivo, dentro de comunidades humanas equitativas e amorosas.

\section{Palavras-chave}

Mulheres indígenas; empregos femininos; cuidado familiar; cuidado da terra; Mãe Terra; chagra

\section{Warmikunamanda antiva warmikunamanda chagpimanda runakuna i sug rimaikuna Maria Joseua}

\section{Maillallachiska}

Kai kilkaskapi churanimi sug rimaskakuna Nukanchipa achala warmikunaua i iachakunaua chagpi Araracuaramanda (Caqueta), i sug warmi i karikuna kaugsagkuna ambi upiaspa chiua kadurkuna i imasami respetankuna kaugsaita: paikuna kauachinkuna imasa kaugsanga sug rigcha occidentalmandasina. Kai regseita kanmi kauachii imasami warmikuna paikunapa iachaiua chagpipi, i sug warmi i karikuna kankuna comprometido sachukua i familiaua, uiñachiska conexión pacha mamaua i cosmos iapa kaugsa kaspa kaugsaspa comunidadua runakunaua pariju kuianakuspa.

\section{Rimangapa Ministidukuna}

Nukanchi pura warmikuna; ruraska,warmikunapa; kauai familiata; kauai pachamamata; Pachamama mikui taarpuska kuar 


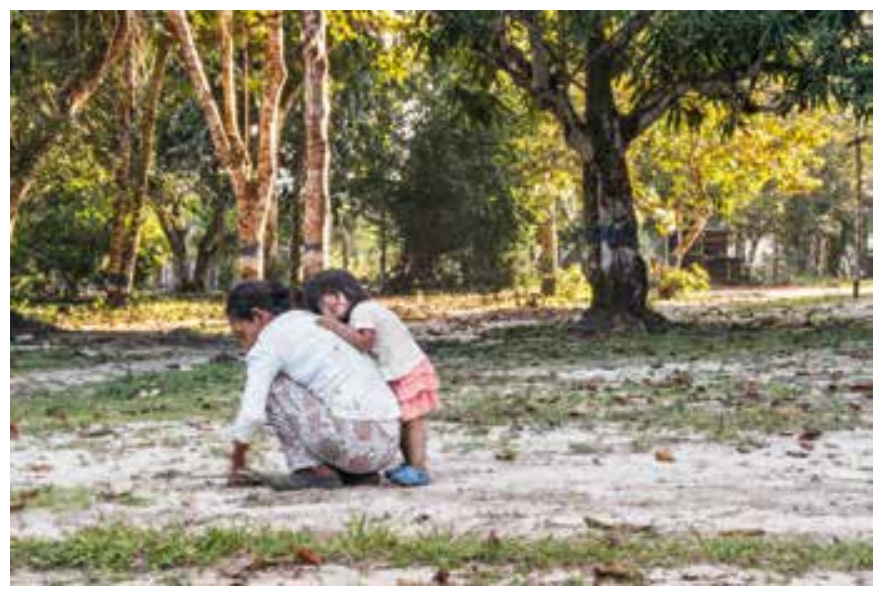

Imagen 1. Abuela y nieta en Villa Azul (Caquetá)

Fotografía de César Vargas.

Introducción: Es tiempo.... Es tiempo de comprender e integrar todo lo que aún sientas que está fuera de ti.

Todo lo que te rodea es la proyección de ese punto de conciencia que percibe que eres.

En los momentos de evolución que vivimos en nuestro planeta los empeños de nuestras almas están enfocados en hacernos crecer y crecer, su objetivo siempre fue que la conciencia que somos volviera a contenerlo TODO.

(Simona, 2011).

Me han atravesado sentires y pensares - la palabra injusticia ha aflorado muchas veces en mis labios (como en el de tantas mujeres) - a raíz de vivencias dadas por nuestra condición femenina en el mundo en que nací. Por otro lado, he compartido palabra, he tejido pensamiento de historias sobre el amor, el cuidar, el sembrar, el criar a los míos y a los suyos de todas las mujeres. Me he preguntado: ¿Será que aprendo a amar, valorar y cuidar la naturaleza, sembrando y preparando pócimas con las hierbitas?, ¿Será que transformo mi pensamiento y creencias ocultas, y los manifiesto a través de la conciencia en rituales cotidianos? ¿Serán estas las vivencias, desde donde tejo mi humanidad?

Este texto une historias presentes y pasadas, contadas en diversos espacios: la casa, la cocina, el camino, la chagra y la maloca; está hecho de diálogos ${ }^{1}$, historias, relatos, chismes, publicaciones y citas bibliográficas.

$1 \quad$ La idea es tener un diálogo de pensamiento entre nosotras, en donde el sentir tiene su forma de expresar.

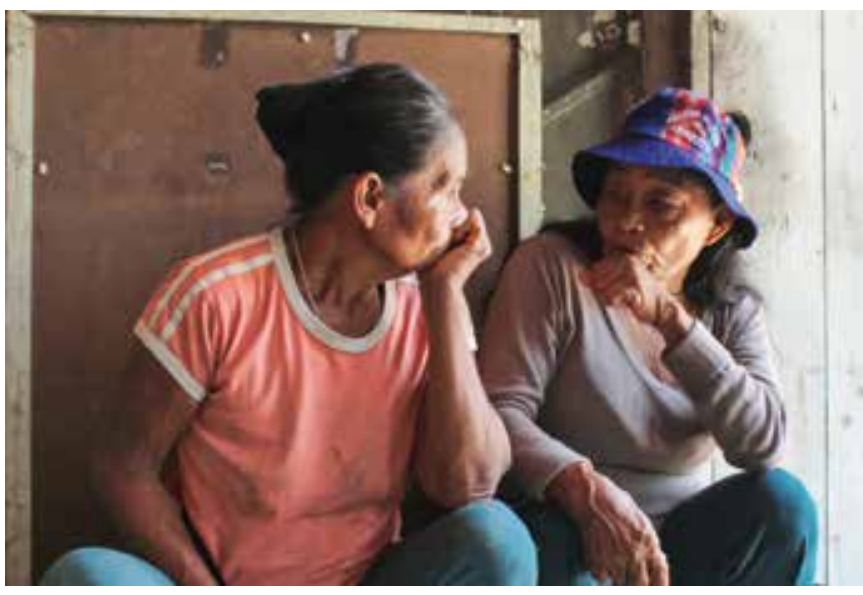

Imagen 2. Las abuelas Juanita y Lucrecia.

Fotografía de María José Arbeláez.

Nazaret Cabrera (2016) hace una reflexión de cómo comparten el conocimiento las mujeres de Gente de Centro ${ }^{2}$, cuando se encuentran entre ellas: uno dice, que aporta desde su vivencia y decimos... pues vamos recogiendo historia, pues cada uno de diferente manera aprendió. ¿Yo que aprendí de mi mamá? ${ }^{3}$ ¿Y mi mamá qué va a dejar?, o entre todos ¿Qué vamos a rescatar? Yo hablo en los relatos lo que ellos me enseñaron, sobre lo que hizo mi mamá o mi papá, esto me decían mis abuelos, todo ese mundo de enseñanza que se comparte con la naturaleza.

Este escrito no se restringe a un sentido univoco, es una articulación heterogénea, polifónica, es un cruce de vivencias y saberes, conocimiento vivo, con sentimientos, reflexión y vida interior, en donde se recogen las experiencias particulares respecto a la condición del ser mujer indígena o mestiza, a la valoración y el sentido creativo de los trabajos domésticos, a las relaciones de las personas y la naturaleza, y al sentido y el compromiso del estar vivos de cada una de las personas con quienes conversé. La figura que evoca es un círculo de palabra. De hecho, alguna palabra o tópico tratado por una persona es ampliado por otra voz, por ello, algunas veces, los textos están incrustados entre sí. Las abuelas con quienes entramos en diálogo fueron: Alicia Sánchez (etnia Uitoto, Minika), Raquel Andoque (etnia Andoque), Marlene Ramírez (etnia Uitoto, Nipode), Alcira Sueroque

$2 \quad$ La comunidad Gente de Centro está conformado por cuatro pueblos indígenas: el pueblo Andoque, el pueblo Nonuya, el pueblo Muinane y el pueblo Uitoto.

3 Desde el momento en que los abuelos comienzan a hablar, nosotras somos las que escuchamos, no debemos interrumpir; se vuelve, pues, el escuchar un accionar introyectivo y comprometido, no para refutar inteligentemente, sino para aprehender y aprender. 


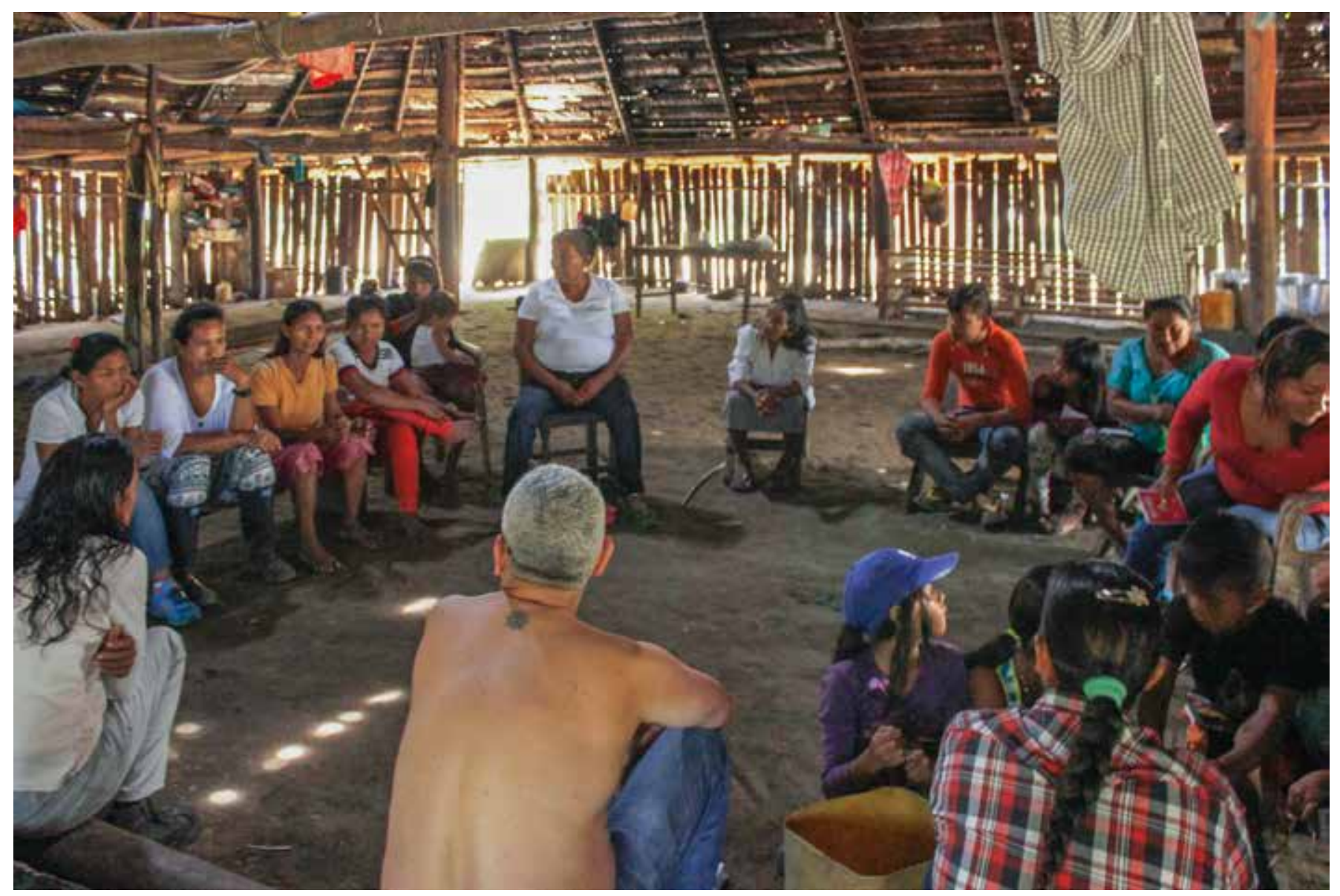

Imagen 3. Recuerdo del taller con las abuelas en Araracuara. Fotografía de César Vargas.

(etnia Uitoto Minika), María Sabina (etnia Muinane Nonuya), Nazaret Cabrera (etnia Uitoto, Nipode), Aidé Barbosa (etnia Muinane), Patricia Márquez (etnia Uitoto, Minika), Juana Suárez (etnia Muinane), Isolina Simón de Cabrera (etnia Uitoto Nipode), Aurora Matapí (etnia Matapi Yukuna), Amalia Gómez (etnia Uitoto, Muinane) y Rufina Román (etia Uitoto); los sabedores Aurelio Suárez e Isaías Román; las escritoras Ximena Noemí Ávila Hernández, Miranda Gray, Clarissa Pinkola, Cristina Carrasco, Vandana Shiva, los escritores Eduardo Galeano y Patricio Guerrero, y el investigador artista Andrés Corredor.

En la primera parte de este escrito, ¿Utopías? Modos de ser y estar en comunidad, las mujeres, discurrimos sobre modos de habitar en la costumbre del "deber ser" femenino, de las cosas en la intimidad de las relaciones, y nos preguntamos por la palabra justicia para con los míos o con los otros, desde historias de violencia física o violencias más sutiles, las que solicitan cambios de ser y estar. En el aparte Apenas cae la pluma blanca: vivir con gracia, conversamos sobre cómo en el vivir cotidiano hay respeto y valoración por los pensamientos, sentimientos y actos que realizamos en tanto seres sagrados, los cuales cohesionan o conforman las comunidades familiares. En Las mujeres cuidan la comida y los hijos, hablamos sobre la mujer trabajadora de chagra en el mundo de la "Gente de Centro", y sobre uno de los rituales de inicio de los trabajos de los hombres, en el entendimiento de que los hombres y las mujeres queremos respetar y cuidar a todos los seres vivos; también, sobre el sentido que le damos a nuestra existencia cuando vivimos conscientemente nuestros actos, actuando como seres coherentes. Finalmente, en Tierras amorosas: calladita, no diga nada, dialogamos sobre el trabajo domestico realizado por las mujeres como conjunto de procesos creativos que fundan un saber, materialismo vitalista femenino ${ }^{4}$ sobre prácticas de cuidado de sí, de los otros y de la naturaleza.

La conversación sobre las mujeres, desde las historias de las mujeres, se da principalmente con las abuelas Gente de Centro en Araracuara, localidad ubicada en el curso medio del río Caquetá entre los departamentos

\footnotetext{
$4 \quad$ Vandana Shiva propone un saber materialismo vitalista acerca del sostenimiento de la vida dada la dedicación de las mujeres a las actividades de sostenibilidad de la vida o trabajos de cuidado.
} 
colombianos de Caqueta y Amazonas, desde la pregunta por los saberes sensibles practicados por las mujeres indígenas Gente de Centro en Araracuara, en relación a los cuidados de la naturaleza y de la familia, mediante el recorrido de sus chagras durante los años 2015 a 2017. En esta investigación, la conversación es un método de conocimiento vivo, invita a reflexionar en que nosotras recibimos y transmitimos las visiones de las abuelas y las nuestras propias, que transformamos nuestras vidas por medio del intercambio de la palabra, del canto, de las acciones, de los rituales y de los sueños que nos afectan y te afectan en la medida en que formamos comunidades comprometidas entre sí y con la vida, la naturaleza y el cosmos.

\section{¿Utopías? De modos de ser y estar en comunidad las mujeres}

La mujer siempre es la madre tierra, porque nosotras somos eso, somos las que damos la vida, no es el hombre; el hombre si aporta, pero dónde está la vida, es en el vientre de nosotras. Cuando sale es lo mismo que cuando una planta retoña de la tierra, las semillas caen; la lluvia con su acompañamiento y el calor, ayudan a fecundar, es por eso que la mujer siempre se está comparando con la tierra, con el cultivo, con sus plantas; por lo mismo, por lo que uno está sembrando, uno es la que está cosechando, uno es la que está limpiando, una la que está recogiendo; siempre hay una comparación con eso de que nosotros somos naturaleza porque nuestro vientre se gesta, para después brindarlo a la humanidad. (Alicia Sánchez: 2015)

Nos enseñan las abuelas sobre la importancia de que como mujeres tomemos conciencia del poder creativo de nuestro vientre, de nuestros ciclos lunares, de las energías que se movilizan y de lo interconectadas que estamos entre nosotras: Anoche soñé tejiendo un cinturón... con diferentes colores rojos... Ximena Nohemí nos cuenta en Relatos del Cántaro (2016): Había llegado el día en que la niña se había hecho mujer, pues la sangre de sus venas había comenzado a bañar su útero. La madre la bendice pintando su rostro en símbolo de celebración, le obsequió joyas y flores, y le da en sus manos una pequeña madeja de hilo rojo. Esta había sido hilada y teñida por ella misma con su sangre menstrual después de recuperar sus ciclos luego de dar a luz a la niña. Ese especial día, la madre le pidió a su hija prometer desenrollar esta madeja hasta el fin de los tiempos, donde generación a generación cada madre le enseñaría a cada hija este legado, transmitiendo así el valor y la responsabilidad con todo el linaje anterior y posterior: todo lo que vivas se registrará en el hilo rojo y por lo tanto será compartido con todo el árbol; todo lo que sufras lo sufrirá el árbol, todo lo que sanes lo sanará el árbol.

Yo me dije, voy a hacer un cinturón con la sangre de mi mes lunar. Necesito conseguir lanas rojas, lo estoy tejiendo. De él van colgados, una imagen de un animal de poder: la cucaracha (necesidad de trabajar la importancia personal, necesidad de confiar en el poder de la humildad) y una pluma (necesidad de aprender la levedad y de fluir con el viento).

Las energías creativas femeninas, nos enseña Miranda Gray, estaban destinadas a las áreas de la supervivencia, la tradición, la belleza, la comprensión, la maternidad, la claridad interior y la sabiduría. La mujer arreglaba su hogar, organizando los espacios y las actividades de una manera armónica, preparando de una forma amorosa y cuidadosa los alimentos necesarios para nutrir a la familia, armonizando su interior a fin de poder establecer un ambiente cordial y próspero para que su familia se sienta segura, reconocida y amada. Ella tenía conocimientos de cultivar la tierra, cocinaba, almacenaba los alimentos y sabía preparar medicinas naturales. La mujer cosía, tejía, fabricaba cestas, y decoraba todos sus productos, creando historias personales que reflejaban órdenes espirituales ${ }^{5}$.

Y ante la insistencia del querer ampliar la pregunta hacia las prácticas cotidianas y comprender ¿qué es ser mujer?, reconocí historias familiares similares a las mías. Rufina Román (2015) nos cuenta: A mí me parecía como que la mujer no tenía mucho valor. Para mí, me parecía que las mujeres éramos unas esclavas. De pronto, grosera, siempre supe que en la casa las mejores presas eran para los hermanos; mataban gallina y para uno era la patita, esa era mi presa, pero para mis hermanos era lo mejor. Yo veía que mi mamá dejaba de comer para darle lo mejor al esposo y a los hijos, pero a uno no; yo siempre, de alguna manera, cuestioné esa parte. Porque yo nunca sentí cariño, ni abrazo de mi papá, siempre estuve acompañada de mi mamá. Eso siempre fue como algo duro para uno. Yo decía: —Eso de ser mujer, ¿Qué? ¿Por qué siempre ellos? ¿Por qué siempre la mejor atención? ¿Por qué siempre

5 Es necesario, sacralizar las acciones, los pensamientos y las obras; sentirnos como hombres y mujeres espirituales con poderes mágicos, vivir con conciencia e intención en lo que estoy realizando. 
les hablaban a ellos lo que me querían decir? A mí no me lo decían, sino se lo decían a mi mamá. Por mi mal comportamiento le pegaban a mi mamá, y si mi mamá entraba a defenderme, le pegaban a mi mamá también.

Y claro, esto de la violencia intrafamiliar que vivimos en diferentes grados todas las mujeres, duele, y duele espantosamente. Me remite a mi cuerpo también, y a mi forma de vivirlo como un "lugar de tránsito" a altas velocidades, depósito de deambulaciones, fugacidad de imágenes y de palabras, ligereza en los sentimientos, sensaciones, también visión de situación. Correr detrás de... en realidad nada me permea porque soy hueca tanto por dentro, como por fuera. ¿Qué violencia es la que rechazamos nosotras? La que vemos ejercer por el otro o la que ejercemos nosotras mismos. La abuela cuenta: "Eso que sufrió ${ }^{6}$ aún lo sufren algunas mujeres, es muy fuerte".

Y en las conversaciones que realicé con las abuelas indígenas, vi dos diferentes clases de rudeza. La física que se realiza ante la mujer hasta agotar y quebrantar su cuerpo y su alma como cuenta la abuela Raquel Andoque (2015). Este era mi esposo. Yo viví con él, y como dijo la paisana, a los tres días me pegó... y yo le dije a mi papá: -Papá, ese hombre que usted me dijo que era bueno, pues me pegó, yo no sé si voy a vivir con él, y mi papá me dijo: -Ya usted vivió con él, y el papá de él es brujo y te puede brujear... pues viva con él... y yo dije: -Bueno.- Así yo viví con él. Me llevaba para el monte y me pegaba; mi papá nunca se daba cuenta.

Y la otra forma de ejercer la violencia gracias a las fuerzas senescentes de una cultura, a fin de favorecer la continuidad de formas establecidas de comportamientos sociales, solicitando la transformación de modos de ser y hacer de la mujer, como es el caso de lo sucedido a Rufina Román (2016): Con la abuela de mi hijo empiezo a sufrir choques de muchas formas. Tengo que ir a un club, y yo - ¿Cómo así que tengo que ir a un club? Entonces ellas me mandaban a peinar... - Usted tiene que llevar la cartera así, sentarse así, el vaso así. Eso me cansó... yo dije, -yo no soy de ese mundo. Acá uno se sienta como caiga, se siente como más libre. Andar todo el tiempo con tacones puntillas, a mí me tocó ponerme todas esas cosas para ser la esposa de ese señor.

$6 \quad$ Eso que sufrió, aún lo sufren en algunos hogares, es muy fuerte. $Y$ viven juntos. ¿Y aún siguen teniendo hijos? Es la pregunta que uno se hacía. Entonces, ¿había amor? En ese tiempo no... uno se quedaba... (Mujeres Gente de Centro).
Esa gente lo coge a uno, porque yo no sabía ni manejar un peso. Yo tenía todo, pero nunca me dio plata. Cuando llegó mi papá, yo quería darles algo, no pude ofrecerles nada. Quería mandarle algo a mi papá y yo no tenía ni un peso. Eso generó en mí algo... bueno, a mí me están sacando... y yo decía - ¿Qué? ¿Será que todas las paisanas que conseguían marido blanco son así?

\section{Apenas cae la pluma blanca: Vivir con Gracia}

La cuestión del Vivir con Gracia, comprende la pregunta de ¿cómo nos relacionamos, aceptamos, amamos a los seres humanos en nuestras pequeñas comunidades familiares y culturales, a todos los seres, desde la ética de convivencia ejemplificada en las historias sobre las chagras de la comunidad indígena de Gente de Centro? Por ello me remito aquí a sus historias. La abuela Juana Suárez nos llevó a su chagra de rastrojo. En un momento del camino, ella dijo: iY aquí está mi chagra! Y para mí esto no era nada diferente a todo lo que venía viendo en el camino: cantidades de árboles, plantas, matas diversas, ni un pasaje señalado... el lugar era de difícil tránsito. Yo me dije: ¿La chagra? ¿La huerta?, como le decimos en la ciudad. En mi imaginario, es sin hojarasca, los árboles van en fila, ordenados métricamente, agrupados por variedades, o preferiblemente grandes extensiones de monocultivos ${ }^{7}$; se controla la plaga mediante químicos, se ve por donde caminar para no pisar ninguna matita.

Esto me cuestiona sobre el modelo de sembrado cultural que tengo en mi memoria, construido de manera diferente. En la ciudad impera el pensamiento científico y técnico del modo adecuado como debe hacerse un cultivo para satisfacer a los compradores que requieren un deber ser de perfección de los frutos del campo cuando van al supermercado. Y en aquella ocasión caminamos por la chagra, la abuela nos va explicando que en su cultura, en la chagra, se siembra todo mezclado y junto.

Se respeta la diferencia, como nos dijo Isaías Román (2016): El cultivo es una variedad de plantas. El sembrío es una hermandad para poder vivir en comunidad, nadie

$7 \quad$ Acá (en Bogotá), a nosotros nos gusta la guerra siempre. Eso se llama monocultivo. Yo sé que la población de los bichos se va a alterar, todos los días nos toca fumigar, me toca estar peleando. Cuando no tenemos amor en nuestra conciencia, en el trabajo, lo que brindamos a la naturaleza es agresividad, lo que brindamos hacia el otro es agresividad; si tuviera amor, brindaría amor, pero como no lo tengo, entonces, brindo lo que tengo: agresividad. (Isaías Román: 2015) 


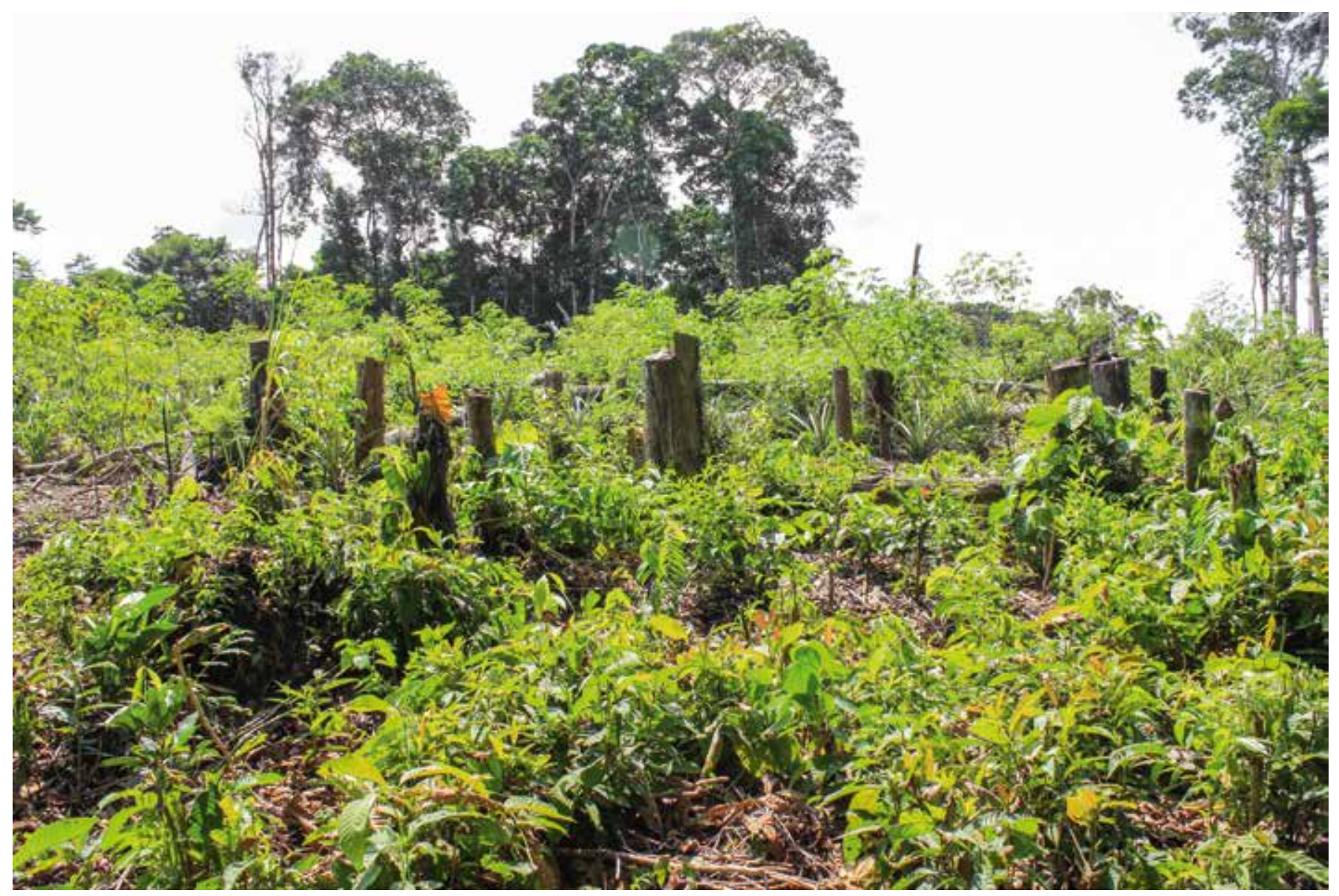

Imagen 4. El sembrío es una hermandad para poder vivir en comunidad. Chagra Cacica Raquel Andoque. Fotografía de María José Arbeláez.

se discrimina. Si usted sembró ají, aquí, nadie le dice: -Ají, quítate de aquí, tú eres picante; aquí está la palma de chontaduro que tiene espina, nadie le está diciendo: -Quítate de aquí, tú eres espinoso. El sembrío en nuestra cultura es una hermandad para poder vivir en comunidad, nadie se discrimina.

Las gentes son diversas: su diversidad es la materia que da lugar al tejido de conocimiento, de la palabra. Todos nos integramos en ese tejido. Así como en el trabajo de la chagra, en donde las plantas son distintas, pero no se discriminan entre sí, sino que por el contrario se protegen mutuamente. En el tejido de los humanos, cada humano, cada grupo, si bien tiene su propio ojo, su propia visión, se reúne en el conjunto de ojos del tejido del canasto, en el que se encuentran los conocimientos.

Y los sabedores y las abuelas nos hablan de que somos seres vivos y estamos interconectados, que las plantas, los tipos de tierra, los animales, las aves, todos los seres vivos, se conectan por su color de clan. Dice Isaías, es dolor y amor, si existe esa ave o gato allá, estamos en familia, si se afectan ellos, nos sentimos todos.
Por ello, la tradición nos dice que en los trabajos que realizamos no podemos hablar sin sembrar nada. Rufina Román nos cuenta: Mi papá nos obliga, nos regaña. Tenemos que ir. Mi papá dice —Usted habla en público ¿Cuál va a ser su fuerza? ¿Cuál va a ser la planta que te va a cuidar desde allá? ¿Quiénes son las que cuando usted esté enferma le van a decir: "Venga, pase parte de la enfermedad" ¿La vamos a recibir nosotros? No tienes fuerza, tienes que sembrar, entonces a uno lo... (No es que lo obliguen), le enseñan a tener el contacto con la tierra, a reemplazar eso que ellos tumbaron... tratar de reemplazar... ¿No ve que nosotros destruimos territorio de un animal? Hay que volver a sembrar para que ellos vuelvan a vivir ahí. Entonces de ahí en las chagras una empieza a notar que [ade]más de que es la mujer la que cuida [que ese es el concepto que se tiene] la chagra es "ariare conmune"8.

Teniendo en cuenta que lo comunitario empieza cuando yo siembro los parientes de las gentes que habitaban el

8 Ariare conmune significa pueblo, gente de frutales o vegetales. 


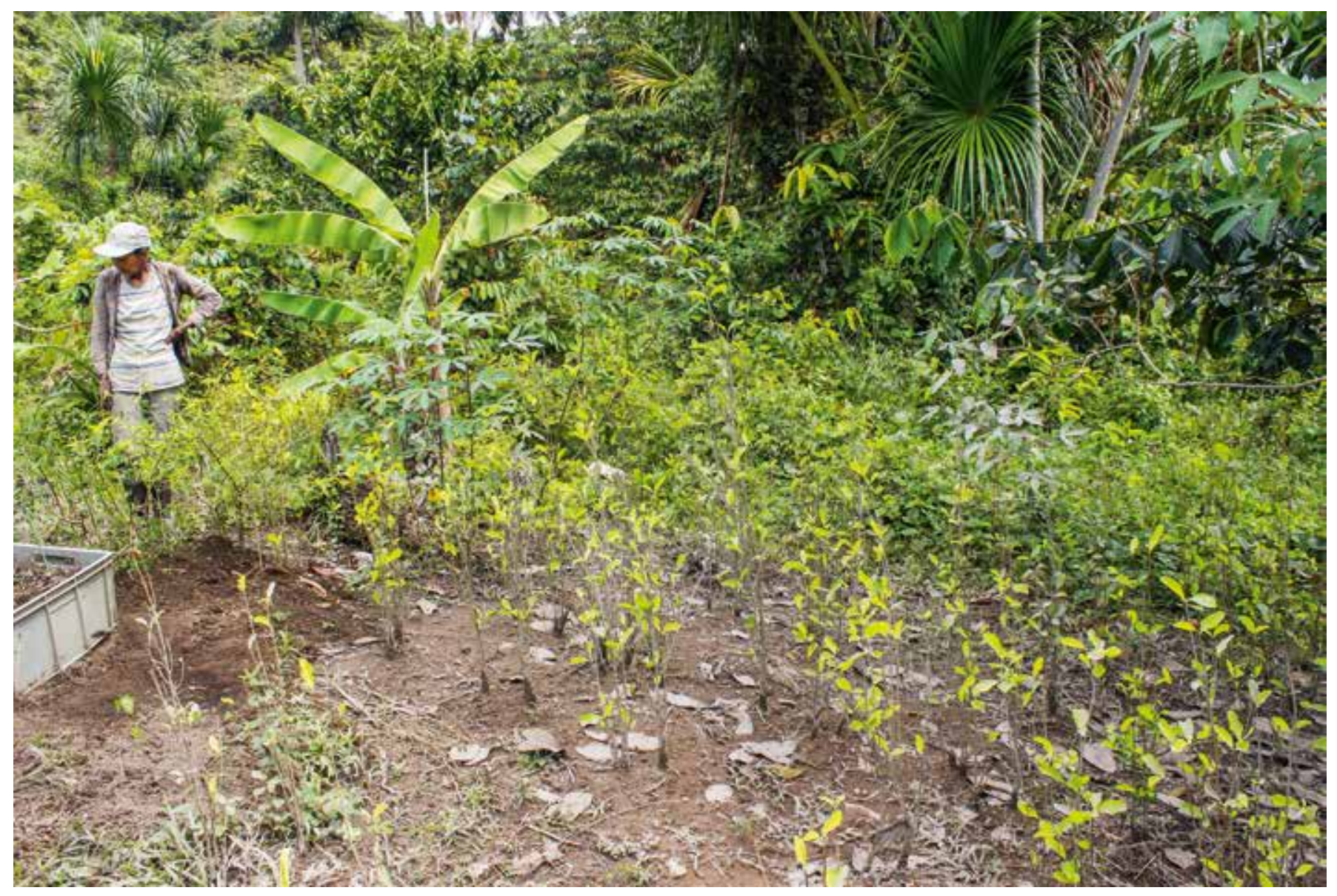

Imagen 5. Chagra de la abuela Marlene Ramírez. Fotografía de María José Arbeláez.

terreno, siembro lo que necesito como medicina en el cuidado de mí mismo, y también siembro para mantener la vida silvestre ${ }^{9}$. No se rompe la conexión, la naturaleza puede regresar. Además de que en la siembra va la liberación, mi curación mediante mi trabajo; lo colectivo que implica vida y salud para todos, explica Nazaret Cabrera (2016).

Como nos cuenta la cacica Raquel Andoque: el ritual que se hizo antes, en su inicio de trabajo hace años atrás, todo usted nombró; nombra los animales, nombra los peces que están en el cultivo, la danta, el zaíno; la maraca es boruga, el maíz es armadillo, las uvas son micos; bueno... así sucesivamente. La chagra es pues, el centro de relaciones sociales, es conocimiento aprender a vivir de tal manera en que soy consciente de que lo que hago afecta a todos, como dice Isaías, estando claro que para la Gente de Centro "Todos somos una

9 Identificar seres. Cuando voy a escoger el terreno para sembrar, no voy solo, me acompañan todas las gentes que habitan el lugar y en diálogo escogemos el lugar. Observo qué es lo que yo desforesté, para así mismo remplazarlo con algo de parentesco, como de familia. (Isaías Román) familia, todos somos sembrados y estamos interconectados: somos seres colectivos".

\section{Las mujeres cuidamos la comida y los hijos}

"La tarea de las mujeres, dar y cuidar la vida en todo su ciclo es imprescindible para la conservación de la especie humana". (Carrasco, 2009)

Es necesario visibilizar la tarea fundante que desarrollamos las mujeres como seres sensibles que nos preocupamos del sostenimiento de la vida y del cuidado de los hijos en el hogar y en la comunidad de seres vivos en general, puntualmente me refiero a las practicas de abuelas y madres de la comunidad Gente de Centro, como las realizadas también por las mujeres de comunidades de las ciudades donde habito.

Aunque nacer mujer es ya de por sí un reto, los padres se sienten traicionados pues quieren continuar su linaje a través de los hombres, las madres felices, aunque sufrimos miedo de toda la violencia familiar $y$ 
social que sobrellevamos por el hecho de nacer mujer. Anteriormente, en las comunidades Gente de Centro, Nazaret Cabrera nos cuenta: Desde ahí mismo, desde el mismo mito ya nos dicen, bueno, ¿Qué vas a tener? Un niño, nooo, porque hay un mito que dice que los niños, que los hombres han sido malos, - te acuerdas que en Araracuara hay un lagartijo pequeño que siempre anda en la casa-, en la chagra también, ellos viven y ellos hacen huecos porque ellos también conviven en la chagra y con el ser humano; entonces qué pasa, que este animalito cuando le decía que va tener un niño, decía no, porque los niños nos tiran piedras, los hombres no nos quieren, ellos son malos, entonces en el momento del parto esa mujer va a sufrir. Que va tener una niña, esa sí las bendiciones, porque la niña va a ir la chagra, va limpiar la chagra, a limpiar donde nosotros dormimos, antes nos cuida, siembra para nosotros, entonces, es una bendición. Por ello se decía, es una bendición cuando nacía una niña, en cambio hoy en día, si nace una niña, como que hay un pero, iAy no!... mejor dicho... van a ser la que hace el dolor de cabeza; la que, mejor dicho, o sea, en este momento, a nosotras nos miran como lo más, cuando anteriormente es una bendición, en cambio hoy en día, somos el dolor de cabeza.

Uno de los trabajos de las mujeres es la enseñanza a sus hijas, a sus nietas. Las mamás enseñan algunos reglamentos a tener en cuenta cuando ya se es muchacha: haga oficio, no duerma, no sea perezosa, porque si usted se va a vivir con su marido usted tiene que estar trabajando, tiene que atender sus niños, entonces usted no puede estarse quieta. Usted tiene que estar en movimiento porque si no, después a usted la deja, se consigue a una mujer trabajadora y usted va a ser la vergüenza de otros, usted va a ser vergüenza de toda una comunidad. Eso era fatal. Por eso era siempre... levántese temprano, báñese la boca, el agua te da fuerzas, no te envejece; si usted se baña temprano no se va a a envejecer, porque si usted se baña después de que la mariposa se ha bañado, de que la danta ha tomado agua, después de que los animales del monte ya se han bañado, eso le trae todos los males a su cuerpo. Usted tiene que levantarse temprano y bañar su cuerpo, por eso es que a ustedes les gusta contestar, son groseros, porque usted se baña a la hora que se bañan todos los

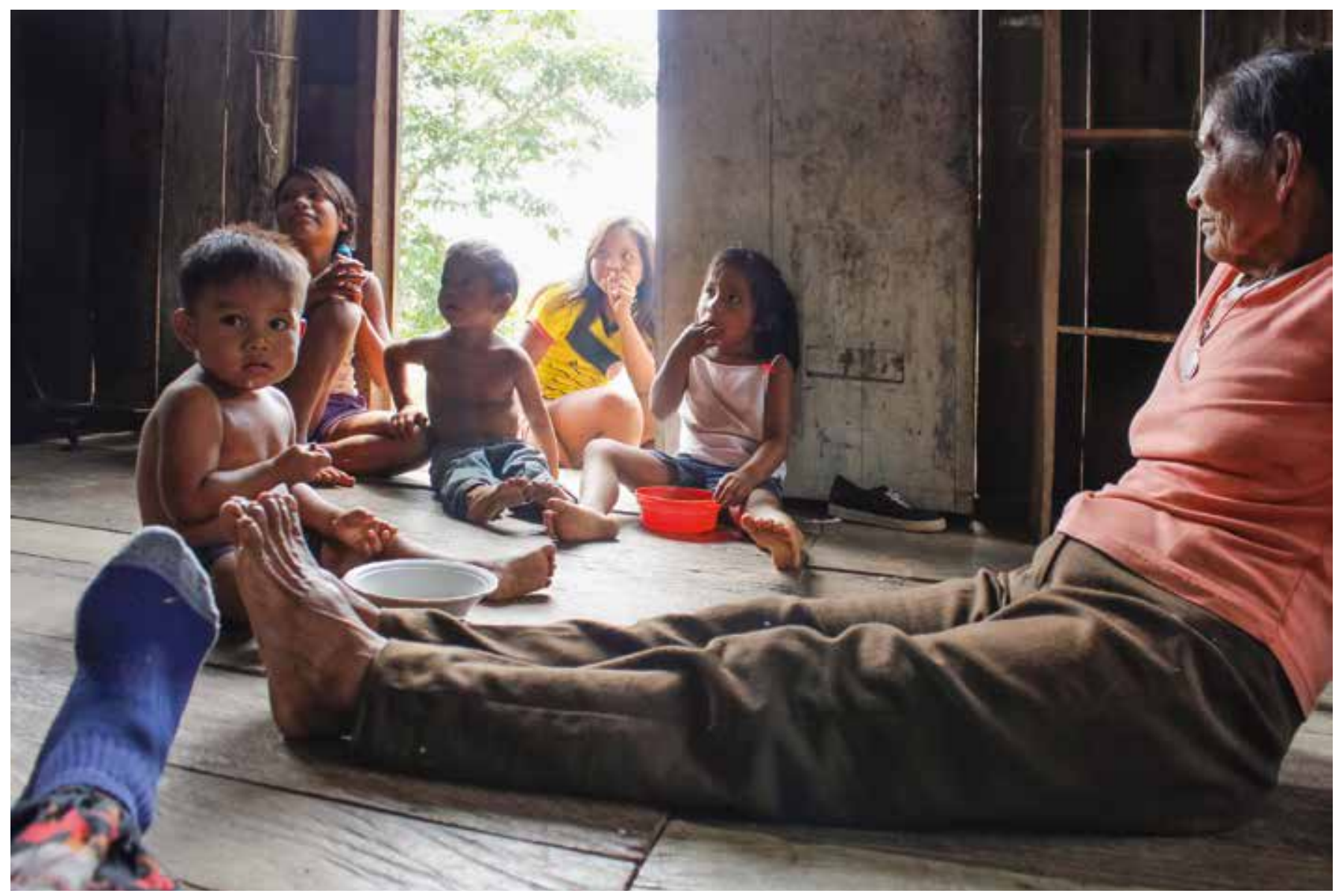

Imagen 6. La abuela Juana cuidando niños de su familia y de vecinos. Fotografía de María José Arbeláez. 
animales; usted no mira cómo los animales son necios, los animales no hacen caso, pelean, se matan, es una serie de comparación con el medio natural, entonces sí... uno tiene que hacer caso.

Las mujeres son mujeres trabajadoras de chagra, nos explica Alicia Román: Ella siembra así, así mamá siembra, va tirando así, va clavando, carga, "moli juaunu": mujer... mujer muy sabedora, trabajadora, no más clavarla: carga; sí, ese es el nombre: "tatanoruno", una mujer tatano, ella es buena con las manos... ella lo siembra así, carga maní, llena esa mano maní, mano ají, ají, maní, o sea madre de la chagra, ese es el nombre. Que crezca, que carga así como ella hacía, así se hace; se reza para que no termine, para que retoñe bien, carga que retoñe, que no sea raquítico, la yuca.

La cocina es trabajo de las mujeres. El orden de la casa lo hacen también las mujeres, el cual implica una serie de actividades materiales como el arreglar, limpiar la caza, cocinar los alimentos, lavar la ropa, los enseres domésticos (fuera del mantenimiento de la chagra). Las actividades de gestión y organización fuera de la casa; como también actividades de relaciones afectivas, de cuidado, de calidad de vida, que no pueden ser cuantificadas ni valoradas en términos únicamente monetarios. Es en esas actividades cotidianas que se dan los patrones de conducta de los individuos y por lo tanto se crea y recrea la cultura.

La sabiduría indígena, como lo relato, reconoce el papel de la mujer como creadora de vida, y co-creadora con el espíritu: fuerza que cohesiona a la comunidad, que convoca, que establece alianzas y equilibra, desde la afectividad, los poderes de la vida. Lo anterior es un simple recordatorio de todos los trabajos que realizamos las mujeres.

A veces, me pregunto sobre mis sentimientos-acciones respecto a la tierra a partir del ejercicio de la tensión sobre los modos como establezco comunicación con los otros mundos en mi vida diaria. En ello es importante la memoria sobre lo vivido y lo caminado, para su reordenamiento de otras maneras, desde las intenciones y un acompañamiento amoroso de la mujer respecto a todas las que han sido sus ancestras. De este modo realizo acciones cotidianas como rituales propios en tiempos presentes: la acción de beber agua - pero conscientemente, esa agua que bebo, ¿sí tiene que ver con mi existencia? Barro, ordeno los objetos de la casa... pero, ¿ello tiene que ver con mi vida? Y si comienzo a vivir conscientemente mi cotidianidad -tiempos lentos que permiten ver la textura de las cosas - y si soy reflexiva de que estoy viva... si yo limpio los platos en mi casa y limpiando esos platos, limpio mi corazón y mi alma... le estoy dando un sentido. Si yo ordeno mi casa, ordenando mi casa, ordeno mi mente... Yo le estoy dando un sentido, porque si no, las cosas que yo hago nada tienen que ver conmigo y al final yo no puedo existir con todo lo que pasa.

\section{Tierras amorosas: calladita, no diga nada}

La primera mujer trabajadora, la que nos trajo a la chagra las primeras semillas, para que no sufriéramos de hambre, nos dejó la enseñanza de mantener y cuidar la unidad familiar. Nuestro hermano sol, cuando estuvo en el mundo, nos dejó la tarea de cuidar y proteger a las generaciones venideras para que no haya sufrimientos, desgracias, para vivir en armonía con nuestro medio a través de la palabra de consejo; para que protegiéramos a nuestra madre tierra dentro del saber-hacer. (Cabrera: 2015)

Isaías Román nos cuenta con nostalgia de su infancia: Antes comíamos allá como familia: papá, mamá, hijos, pues tienen la misma chagra para todos, se llama como familia. - Hogar es diferente, digo: —Van a nacer los hijos y cuando ya nacen los hijos ya desaparece... esa llamada pereza o cansancio, desaparece... porque por amor a ellos tengo que trabajar; para brindarle a ellos tengo que hacer eso ¿Se da cuenta entonces? Ya esa comida tiene propósito, intención, amor. Entonces para no comer comida estrellada... pues la sembró con pereza, la sembró malgeniado... entonces la persona come esa comida, eso es lo que recibe; para no llegar hasta allá, la chagra regula muchas cosas, pues hasta ahí es para la familia.

Esta practica amorosa cotidiana del cuidado familiar la hacemos las mujeres cuando habitamos desde la atención, para la transformación de mí, de ti, de nuestro existir por actos ejercidos durante muchos años de amor expresado en la creación de un hogar ${ }^{10}$ que

\footnotetext{
10 La mujer uitoto es muy callada, callada hace sus cosas sola, no manda... calladita saca leña, calladita saca agua, calladita hace lo que hay en la cocina, prende la candelita, hace casabe, caguana, manicuera... de los productos que haya en la chagra... los rastrojos en el monte, que uno va y saca pepas de monte; usted cuadra su día de trabajo normal, como mujer, en la medida de lo posible ella hace sus cosas sola, ya que si el señor quiere colaborar, van a ir los dos a pescar... que van a ir a sembrar los dos. Ya es porque le nace al esposo hacer eso. (Rufina Román: 2016)
} 


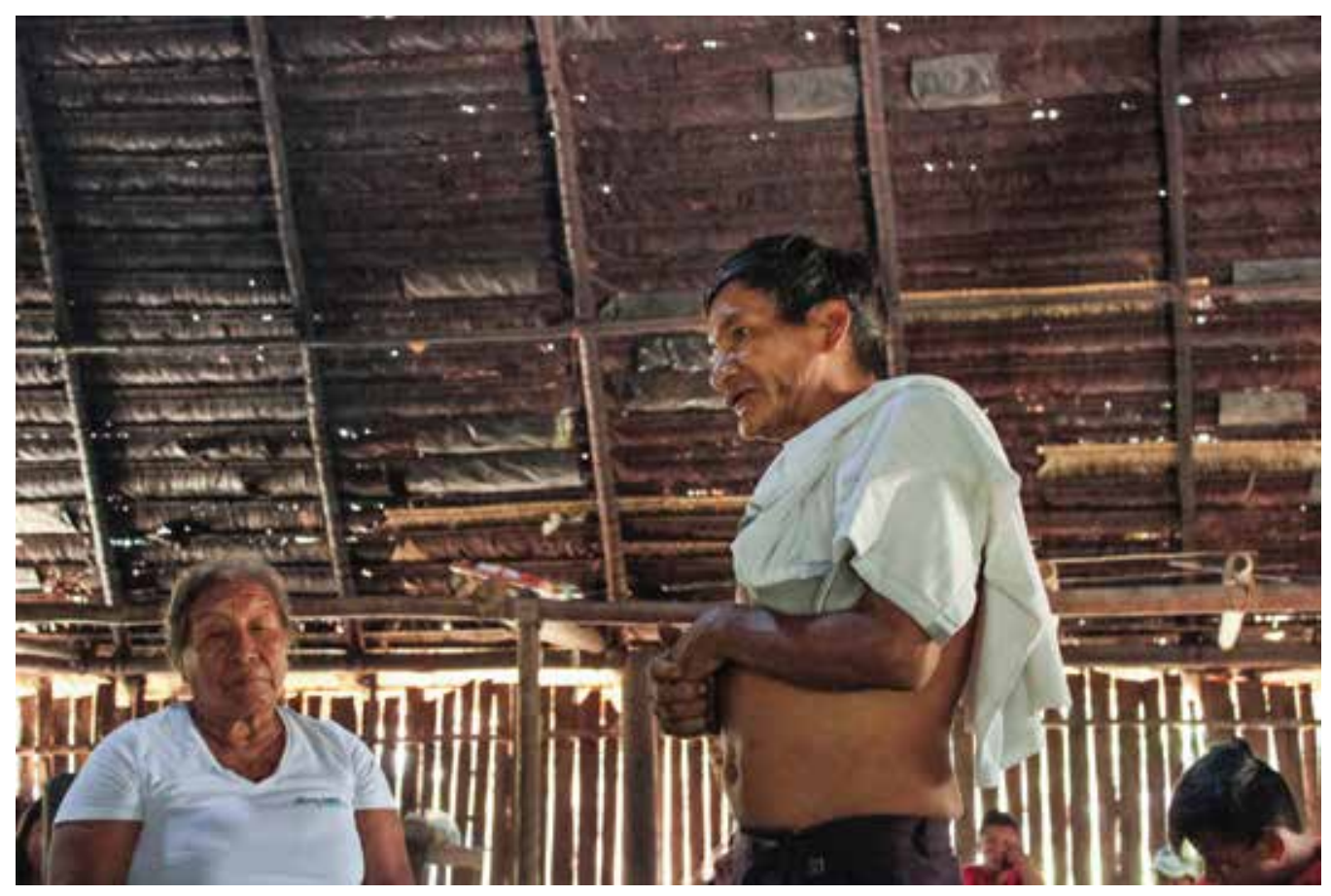

Imagen 7. El sabedor Aurelio contando historias de Chagra. Fotografía de César Vargas.

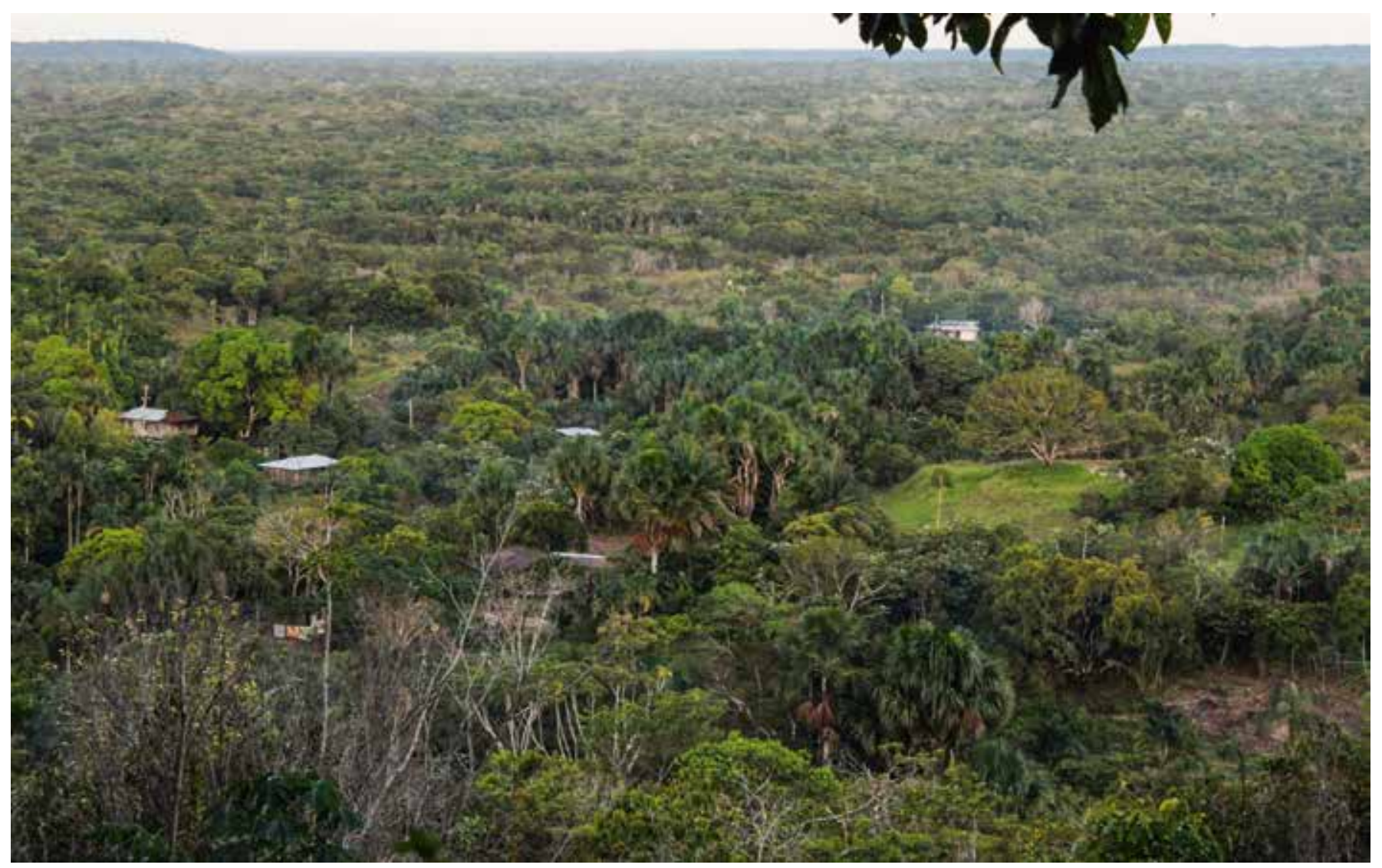

Imagen 8. Vista de Araracuara, Caquetá. Fotografía de César Vargas. 
da seguridad, afecto y calorcito del bueno; son actos creativos, hechos desde y con sensibilidad, intuición: el sabor de la comida, el canto del agua, la brisa que limpia, la risa de niños y la alegría de vivir con los animales, en la conformación de Tierras amorosas.

Como lo señala Vandana Shiva (2006), las mujeres muestran con sus vidas que el amor y la compasión, compartir y dar, no solo son cualidades humanas posibles, sino que constituyen cualidades necesarias para que seamos humanos. Por ello es prioritario debatir sobre el concepto, el significado y las vivencias que se dan en torno al trabajo doméstico, este debe ser analizado desde un cambio simbólico de paradigma cultural importante: "el centro de los objetivos sociales, políticos y económicos (de la sociedad) debería ser la vida humana y las diversas actividades deberían girar en torno a este objetivo primero y estar a su servicio" (Amoroso et al. 2003: 10). Es decir, que el ámbito doméstico para ser analizado en su real importancia, debe recibir una mirada diferente a la que le da la sociedad capitalista patriarcal.

En las lógicas del mercado capitalista el estudio de la economía y la industria se centra en la maximización de la rentabilidad con el mínimo esfuerzo y tiempo. Como indica Cristina Carrasco (2009), se identifica lo económico con lo mercantil, la llamada eficiencia económica aparece estrechamente vinculada a un conjunto de procesos de racionalización y de 'ahorro' de tiempo. El tiempo se considera un 'recurso escaso' con características de homogeneidad, que permite reducir su tratamiento a términos de simple cantidad. El tiempo se convierte así en algo cronometrado, en tiempo-reloj, un tiempo objetivo medible en unidades físicas. En esta lógica las actividades domésticas 'roban tiempo' a la producción.

Otro de los problemas que acontecen alrededor del tiempo de trabajo en la sociedad industrial pone de manifiesto la tensión existente alrededor del orden de importancias: la obtención de beneficios o el cuidado de la vida humana. Esto hace visible los intereses prioritarios de la producción capitalista, los cuales no contemplan el trabajo de la reproducción y sostenibilidad humana y optan por la producción y la obtención de beneficios, aunque ello vaya en demérito de la calidad de vida de las personas. En pocas palabras, al sistema económico capitalista no le interesan los individuos, ni la consecución de una buena calidad de vida. Los costes de trabajos de reproducción de la vida humana se asumen al interior de los hogares, los cuales repercuten en su economía, como en la calidad de vida de los miembros de la familia, especialmente de las mujeres y de los niños. Las mujeres deben realizar conciliaciones entre ambos mundos permanentemente, lo cual hace visible la injusticia para con ellas, en relación con los tiempos y trabajos, mostrando las desigualdades entre géneros, lo que conlleva a una vida en estrés y enfermedades físicas y psíquicas (Amoroso et al., 2003).

Estamos entablando, pues, una conexión profunda entre la sostenibilidad de la vida (doméstica) con la sostenibilidad social y ecológica que implica una relación armónica entre la humanidad y la naturaleza, que es imposible si no se dan relaciones de equidad e inclusión. Por ello le apostamos a que en los procesos humanos, en los proyectos culturales, se tejan relaciones de interdependencia y de integridad en comunidad con todos, unido a la apuesta ética por la vida realizada por las mujeres como ética de la Tierra, esto último siguiendo la invitación de la filósofa Vandana Shiva.

\section{Cerrando el círculo de palabra}

Las abuelas y los sabedores indígenas de la Gente de Centro movilizan oralmente el conocimiento a través de la narración de historias, mitos, cuentos, y la realización de bailes y demás rituales; sus saberes son un acumulado de experiencia y de sabiduría agenciado mediante el lenguaje metafórico. Estas enseñanzas están ligadas con los lugares donde se habita y con las experiencias corporales, y se orientan desde el cuidado de sí y de la comunidad, al conocer el complejo de las relaciones entre los seres; siendo esta una construcción a través de los tiempos, que siempre es colectiva. Los saberes son vivos y son herramientas de transformación, están en permanente cuestionamiento de las personas y las cosas, como me especifica Andrés Corredor (20152017). Con ellos y con los demás hombres y mujeres bellos y sabios que viven en las ciudades y en otras comunidades con los que tuve la fortuna de dialogar, señalamos la importancia de reconocer el poder de la mujer como dadora de vida biológica y creativa, siempre en armonía con la ciclicidad de la venerada tierra y los ritmos lunares. Es urgente oponernos a la violencia física y social, a la violencia sutil que impone transformaciones en los modos de ser y estar de las mujeres. En segundo lugar, queremos vivificar el respeto, la tolerancia y, aún más, la valoración de nuestras diferentes humanidades, gracias al aprendizaje del modo de cultivar las plantas en la chagra de las mujeres de Gente de Centro. También es necesario visibilizar nuestro 
trabajo como mujeres que cuidamos a la familia, dentro de lo cual esta implícito la enseñanza de valores, de modos de ser sensibles y empáticos con las comunidades de seres con quienes habitamos en todos los mundos, siempre siguiendo el ejemplo de la naturaleza. Y por ultimo, está la alegría profunda que sentimos las mujeres viviendo en esta tierra amorosa desde una relación de cuidado y armonía, basada en la equidad con las comunidades con las que nos relacionamos.

\section{Referencias}

Andoque, Raquel (2015). Entrevistas realizadas en el Primer Encuentro con las abuelas Gente de Centro. Araracuara.

Arbeláez Grundmann, M. J. (2018). El arte de andar en la naturaleza. Calle 14 Revista De investigación En El Campo Del Arte, 13(23), 170-185. https://doi. org/10.14483/21450706.12997

Ávila Hernández, Ximena Noemí (2016). Cántaro Sagrado. La madeja de hilo rojo que une a las mujeres. Disponible en: http://www.cantarosagrado. cl/2016/02/14/la-leyenda-del-hilo-rojo/

Cabrera, Nazaret. (2015-2016). Entrevistas realizadas en el Primer Encuentro con las abuelas Gente de Centro. Araracuara.

Carrasco, Cristina. (2001). La sostenibilidad de la vida humana: ¿Un asunto de Mujeres?. Publicado en la Revista Mientras tanto, N. 82. Editorial Icaria. Barcelona.

(2009). Tiempos y trabajos desde

la experiencia femenina. Papeles de relaciones ecosociales y cambio global. No 108. Barcelona.

Corredor, Andrés (2015-2017). Diálogos realizados durante la investigación El saber sensible del entorno de las mujeres indígenas Uitoto, Caminando las chagras personales, familiares y comunitarias en Araracuara, localidad del Amazonas.
Galeano, Eduardo (2013). [La Nueva República]. Recuperado de: https://www.youtube.com/ results?search_query=eduardo+galeano+mujeres

Guerrero Arias, P. (2011). Corazonar el sentido de las epistemologías dominantes desde las sabidurías insurgentes, para construir sentidos otros de la existencia (primera parte). Calle 14 Revista De investigación En El Campo Del Arte, 4(5), 80-95. https://doi.org/10.14483/21450706.1205

Guerrero, Patricio. (2011): Corazonar la dimensión política de la espiritualidad y la dimensión espiritual de la política. Alteridad 10. Revista de Ciencias Humanas, Sociales y educación, Universidad Politécnica Salesiana del Ecuador. https://doi.org/10.17163/alt.v6n1.2011.02

Gray, Miranda. (1995) La luna roja. Los dones del ciclo menstrual. Gaia Ediciones. Madrid.

Pauccar, Nicolás. (2015) Así habla un Q’ero. Recuperado de: https://www.youtube.com/watch?v=QMmPTs4V9Y8

Pinkola Estés, Clarissa. (1998) Mujeres que corren con los lobos. Barcelona: Ediciones B, S.A.

Román, Isaías (2015-2016). Entrevista con Andrés Corredor, María José Arbeláez. Bogotá.

Román, Rufina (2015-2016). Primer Encuentro con las abuelas Gente de Centro. Araracuara.

Sánchez, Alicia (2016). Primer Encuentro con las abuelas Gente de Centro. Araracuara.

Simona, Marivi (2011). Círculo de Espejos: Mensaje de la cucaracha. Disponible en: http://creavidablog.blogspot. com/2011/08/circulos-de-espejos-mensaje-de-las.html

Shiva, Vandana (1995). Abrazar la vida. Mujer, ecología y desarrollo, Madrid: horas y HORAS.

Suárez, Juana (2015). Primer Encuentro con las abuelas Gente de Centro. Araracuara. 